\section{Swept-source and optical coherence tomography angiography in patients with X-linked retinoschisis}

N Padrón-Pérez ${ }^{1}$, J Català-Mora'1,2, J Díaz²,3, $\mathrm{L}_{\text {Arias }}{ }^{1}$, J Prat $^{2}$ and JM Caminal ${ }^{1}$

\begin{abstract}
Purpose To explore the structural features of juvenile X-linked retinoschisis (XLRS) using swept-source-optical coherence tomography (SS-OCT) and optical coherence tomography angiography (OCT-A).

Design Retrospective, observational cross-sectional study.
\end{abstract}

Patients and methods Nine patients (18 eyes) diagnosed with juvenile XLRS were included. SS-OCT and OCT-A were used to evaluate the characteristics of the inner/outer retina and the choroid.

Results SS-OCT showed that the inner nuclear layer (INL) was the most commonly affected area (16/18 eyes; $89 \%$ ). No significant differences in central macular thickness (CMT) or subfield choroidal thickness (SFCT) were evidenced between eyes (CMT: $364 \mu \mathrm{m}$ in the right eye $v s 320 \mu \mathrm{m}$ in the left eye; SFCT: 305 vs $307 \mu \mathrm{m} ; P=0.895)$. Best-corrected visual acuity (BCVA) did not correlate with CMT (rs $=-0.19 ; P=0.445$ ) or SFCT (rs $=0.06 ; P=0.795$ ). BCVA was significantly correlated with the following defects: outer plexiform layer (OPL; $\mathrm{rs}=0.50 ; P=0.036$ ); external limiting membrane (ELM; rs $=0.65$; $P=0.003)$; ellipsoid portion of inner segment (EPIS; rs $=0.67 ; P=0.002)$; and the cone outer segment tips (COST; rs $=0.69 ; P=0.001$ ). Schisis at the INL revealed a spoke-like pattern in the foveal region and a reticular pattern in the parafoveal region on en-face imaging. In cases in which the schisis affected the OPL, multiple polygonal hyporeflective cavities were observed in the foveal region.

Conclusions The hyporeflective spaces on SS-OCT were primarily located at the INL and OPL. BCVA did not correlate with CMT or SFCT; however, ELM, EPIS, and COST defects were significantly correlated with worse BCVA. There was a positive correlation between age and SFCT.

Eye (2018) 32, 707-715; doi:10.1038/eye.2017.281; published online 5 January 2018

\section{Introduction}

$\mathrm{X}$-linked juvenile retinoschisis (XLRS) is a rare inherited degenerative disease affecting almost exclusively males. The estimated prevalence ranges from 1 in 5000 to 1 in $20000 .^{1}$ XLRS is linked to mutations in the RS1 gene located at Xp22. ${ }^{2,3}$

Previous reports have evaluated the splitting of the ganglion cell and nuclear layers in patients with XLRS using optical coherence tomography (OCT). External limiting membrane (ELM), ellipsoid portion of the inner segments (EPIS)/inner segment outer segment junction line, cones outer segment tips line (COST)/ interdigitation zone, and choroidal thickness have been also evaluated to explain the progressive visual loss in these patients. ${ }^{4-7}$ However, the evaluation of inner/outer retina and choroid characteristics with the use of newer technologies as swept-source (SS-OCT) and OCT angiography (OCT-A) is very limited. SS-OCT provides faster and higher-quality scans than conventional spectral domain OCT, and these advantages allow to obtain useful information to understand better an uncommon disease as XLRS.

The purpose of this study was to evaluate the features of the inner/outer retina and the choroid in XLRS patients using SS-OCT and OCT-A.
${ }^{1}$ Department of Ophthalmology, Bellvitge University Hospital, Barcelona, Spain

${ }^{2}$ Department of Ophthalmology, Sant Joan de Deu Hospital, Barcelona, Spain

\section{${ }^{3}$ Department of} Ophthalmology, Sant Pau Hospital, Barcelona, Spain

Correspondence: N Padrón-Pérez, Department of Ophthalmology, Bellvitge University Hospital, Feixa Llarga, s/n, L'Hospitalet de Llobregat, Barcelona 08907, Spain

Tel: +34 9326076 00; Fax: 34932607533. E-mail: noelpdrn@gmail. com

Received: 29 April 2017 Accepted in revised form: 2 October 2017 Published online: 5 January 2018 


\section{Materials and methods}

\section{Study design}

This was a retrospective, observational cross-sectional study. The study was approved by the Institutional Ethics Committee and Review Board at Sant Joan de Deu Hospital (Barcelona, Spain).

Patient selection was performed by reviewing the clinical records of patients with a confirmed diagnosis of XLRS (retinoschisis gene (RS1) mutation) at Sant Joan de Deu Hospital between January 2000 and September 2010. Eighteen eyes (nine patients) were included in the study. The following variables were recorded: age; bestcorrected visual acuity (BCVA) measured in Snellen 20feet with conversion to the logarithm of minimum angle of resolution $(\log \mathrm{MAR})$, and spherical equivalent. All eyes included had not been treated by any complication of the disease for at least during a year before performing both SS-OCT and OCT-A. The images of each patient were taken in the last visit.

\section{OCT measurements}

OCT scans were obtained with the Triton SS-OCT (Topcon Corporation, Tokyo, Japan). OCT-A $4.5 \times 4.5$ and $6 \times 6 \mathrm{~mm}$, three-dimension OCT macular cube, and 12 radial B-Scan were performed in all eyes with XLRS. Retinal and choroidal thickness were automatically calculated and presented as a topographic map with nine subfields as defined by the Early Treatment Diabetic Retinopathy Study (ETDRS)-style grid. The nine automatically calculated ETDRS subfields included the following: central subfield thickness (defined as central macular thickness (CMT) for retina and subfield choroidal thickness for choroid) in the inner ring; nasal parafoveal; superior parafoveal; temporal parafoveal; inferior parafoveal; nasal perifoveal; superior perifoveal; temporal perifoveal; and inferior perifoveal, which were surrounded by rings 1,3 , and $6 \mathrm{~mm}$ in diameter.

The OCT software program segments the retina by delineating the boundary between the internal limiting membrane and the retinal pigment epithelium (RPE) layers. Choroidal thickness is defined as the thickness from Bruch's membrane to the inner scleral border. The automated scans were reviewed and any inaccuracies were corrected manually. All measurements were performed at the same time of day.

Schisis in the retinal nerve fiber layer, ganglion cell layer (GCL), inner nuclear layer (INL), outer plexiform layer (OPL), and outer nuclear layer (ONL) were evaluated using vertical and horizontal central SS-OCT and en-face OCT. Multiple en-face OCT images were obtained according to the segmentation level and to the presence of schisis cavities (GCL, INL, OPL, and ONL).
Abnormalities in the outer retinal layers, including the ELM, EPIS, and COST lines, were also checked using vertical and horizontal central SS-OCT scans.

\section{Statistical analysis}

We obtained all measurements for statistical analysis from the last visit (cross-sectional study). The clinical and demographic data for patients were obtained from a database file and subsequently exported to the IBM SPSS Statistics (v. 23.0) program for Windows (SPSS Inc., Chicago, IL, USA). Descriptive analysis was performed, and measures of central tendency (mean and median) and dispersion (SD and range) were determined, as appropriate depending of the distribution of the variable (Shapiro-Wilk test). The Mann-Whitney $U$-test was used to compare medians (spherical equivalent, and macular and choroidal thicknesses) between the right (OD) and left eyes (OS). Correlations between BCVA (logMAR) and CMT, subfoveal choroidal thickness (SFCT), GCL, INL, OPL, ONL, ELM, EPIS, and COST were assessed by the Rho Spearman's rank correlation test. Spearman's test was also used to determine correlation between CMT, SFCT, and age, and spherical equivalent with SFCT. Values of $P \leq 0.05$ were considered statistically significant.

\section{Results}

XLRS was bilateral in all nine of the patients (18 eyes). Median patient age was 13 years (range, 8 to 21). The median refractive error was $0.65 \mathrm{D}$ in $\mathrm{OD}$ (range, -3.75 to $5.25)$ and $0.0 \mathrm{D}$ (range, -4.38 to -6.0$)$ in OS $(P=0.790$; Table 1).

No differences in logMAR BCVA were observed between OD (median 1.0; range 0.0-1.4) and OS (median 0.5 ; range $0.2-1.0 ; P=0.980$; Table 1$)$. In 16 eyes $(89 \%)$, both foveoschisis and peripheral retinoschisis were present. Peripheral retinoschisis without foveoschisis was observed in one eye $(5.5 \%)$ and foveoschisis without peripheral involvement in one eye.

No differences between the eyes in terms of CMT and SFCT were evidenced: $364 \mu \mathrm{m}$ in OD vs $320 \mu \mathrm{m}$ in OS for CMT and 305 vs $307 \mu \mathrm{m}$ for SFCT ( $P=0.895$ for both measurements; Table 1). We also evaluated retinal and choroidal thicknesses in the parafoveal (nasal, temporal, superior, and inferior) and perifoveal (nasal, temporal, superior, and inferior) zones (Table 2). SFCT and age were positively correlated ( $r s=0.663 ; P=0.003$ ); however, SFCT and the spherical equivalent were not correlated (rs $=0.319 ; P=0.197)$.

BCVA (logMAR) was not correlated with CMT ( $\mathrm{rs}=$ $-0.19 ; P=0.445)$ or SFCT ( $r s=0.06 ; P=0.795)$. However, BCVA (logMAR) was significantly correlated with defects 
Table 1 Clinical and optical coherence tomographic characteristics of patients with $X$-linked retinoschisis

\begin{tabular}{|c|c|c|c|c|c|c|}
\hline Patient No./eye & Age (years) & Spherical equivalent $(D)$ & $B C V A \log M A R$ & BCVA Snellen 20-feet & $C M T(\mu m)$ & $\operatorname{SFCT}(\mu m)$ \\
\hline 1 & 8 & & & & & \\
\hline OD & & 0.25 & 1.22 & $20 / 320$ & 179 & 213 \\
\hline OS & & 1.00 & 1.0 & $20 / 200$ & 184 & 230 \\
\hline 2 & 11 & & & & & \\
\hline OD & & 4.25 & 1.3 & $20 / 400$ & 423 & 305 \\
\hline OS & & 6.00 & 0.4 & $20 / 50$ & 361 & 306 \\
\hline 3 & 12 & & & & & \\
\hline OD & & 0.00 & 0.0 & $20 / 20$ & 262 & 286 \\
\hline OS & & 0.00 & 0.5 & $20 / 63$ & 251 & 278 \\
\hline 4 & 13 & & & & & \\
\hline OD & & -3.75 & 0.7 & $20 / 100$ & 414 & 308 \\
\hline OS & & -4.38 & 0.5 & $20 / 63$ & 438 & 239 \\
\hline 5 & 13 & & & & & \\
\hline OD & & -2.00 & 1.1 & $20 / 250$ & 168 & 247 \\
\hline OS & & -1.00 & 0.2 & $20 / 32$ & 318 & 366 \\
\hline 6 & 14 & & & & & \\
\hline OD & & 5.25 & 1.0 & $20 / 200$ & 323 & 405 \\
\hline OS & & 6.00 & 0.7 & $20 / 100$ & 496 & 403 \\
\hline 7 & 16 & & & & & \\
\hline OD & & -0.38 & 0.5 & $20 / 63$ & 415 & 261 \\
\hline OS & & -0.25 & 0.7 & $20 / 100$ & 425 & 316 \\
\hline 8 & 19 & & & & & \\
\hline OD & & -2.00 & 1.4 & $20 / 500$ & 480 & 320 \\
\hline OS & & -2.00 & 0.7 & $20 / 100$ & 320 & 307 \\
\hline 9 & 21 & & & & & \\
\hline OD & & 4.25 & 0.5 & $20 / 63$ & 364 & 420 \\
\hline \multirow[t]{2}{*}{ OS } & & 4.13 & 0.2 & $20 / 32$ & 271 & 358 \\
\hline & & Median (range) & Median (range) & Median (range) & Median (range) & Median (range) \\
\hline $\mathrm{MCT}$ & 13 (8 to 21$)$ & & & & & \\
\hline OD & & $0.65(-3.75$ to 5.25$)$ & $1.0(0.0$ to 1.4$)$ & $20 / 200(20 / 500$ to $20 / 20)$ & 364 (168 to 480$)$ & 305 (213 to 420$)$ \\
\hline OS & & $0.0(-4.38$ to 6.0$)$ & $0.5(0.2$ to 1.0$)$ & $20 / 63(20 / 200$ to $20 / 32)$ & 320 (184 to 496$)$ & 307 (230 to 403$)$ \\
\hline
\end{tabular}

Abbreviations: BCVA, best-corrected visual acuity; CMT, central macular thickness; MCT, measures of central tendency; OD, right eye; OS, left eye; SFCT, subfield choroidal thickness.

at the OPL (rs $=0.50 ; P=0.036), \operatorname{ELM}(\mathrm{rs}=0.65 ; P=0.003)$, EPIS (rs $=0.67 ; P=0.002)$, and COST (rs $=0.69 ; P=0.001$ ).

During follow-up, three patients presented macula-off and peripheral retinal detachments (OD in case No. 2 and No. 5, and OS in case No. 8), which were treated with 23gauge pars plana vitrectomy (PPV) and silicon oil tamponade. Although in these cases the macula was affected, both SS-OCT and OCT-A were performed after silicone oil removal. Case No. 1 developed significant macular and choroidal atrophy in both eyes.

SS-OCT showed that the most commonly affected layer was the INL (16 eyes, 89\%), followed by the OPL (13 eyes,
$72 \%$ ) and ELM (13 eyes, 72\%). Schisis in GCL was present in only 2 eyes $(17 \%)$. The OCT characteristics of the eyes are presented in Table 3.

The SS-OCT showed a spoke-like pattern on en-face mode in all XLRS eyes with foveal schisis at INL and a reticular pattern in the parafoveal region (Figures 1 and 2). When the segmentation was performed at the OPL, enface OCT images showed multiple polygonal hyporeflective cavities in the foveal region with hyperreflective partitions in the parafoveal region (Figure 2). OCT-A did not reveal significant findings. The presence of multiple hyporeflective spaces at the INL 
Table 2 Macular and choroidal thickness measurements in patients with X-linked retinoschisis excluding central macular and subfoveal choroidal thickness

\begin{tabular}{|c|c|c|c|c|c|c|}
\hline \multirow[t]{3}{*}{ Zones } & \multirow{2}{*}{$\frac{\text { Macular thickness map }}{O D}$} & \multicolumn{5}{|c|}{ Choroidal thickness map } \\
\hline & & OS & & $O D$ & OS & \\
\hline & Median (range) & Median (range) & P-value ${ }^{1}$ & Median (range) & Median (range) & P-value \\
\hline \multicolumn{7}{|l|}{ Nasal $(\mu m)$} \\
\hline Parafoveal & $311(163-370)$ & $330(170-375)$ & 0.354 & $250(172-392)$ & $264(175-340)$ & 0.566 \\
\hline Perifoveal & $280(128-312)$ & $284(142-384)$ & 0.353 & $190(136-339)$ & $200(163-298)$ & 0.377 \\
\hline \multicolumn{7}{|l|}{ Temporal $(\mu \mathrm{m})$} \\
\hline Parafoveal & $308(157-360)$ & $302(167-615)$ & 0.825 & $282(190-413)$ & $282(150-368)$ & 0.825 \\
\hline Perifoveal & $265(148-288)$ & $258(149-549)$ & 0.965 & $248(189-402)$ & $270(157-319)$ & 0.401 \\
\hline \multicolumn{7}{|l|}{ Inferior $(\mu m)$} \\
\hline Parafoveal & $288(168-456)$ & $310(160-524)$ & 0.310 & $268(189-402)$ & $279(178-370)$ & 0.757 \\
\hline Perifoveal & $272(152-570)$ & $270(158-450)$ & 0.930 & $247(157-400)$ & $270(15-540)$ & 0.427 \\
\hline \multicolumn{7}{|l|}{ Superior $(\mu m)$} \\
\hline Parafoveal & $313(169-406)$ & $327(172-410)$ & 0.566 & $307(177-372)$ & $286(170-363)$ & 0.895 \\
\hline Perifoveal & $262(134-335)$ & $278(147-361)$ & 0.401 & $277(151-337)$ & $281(156-351)$ & 0.757 \\
\hline
\end{tabular}

Abbreviations: OD, right eye; OS, left eye. ${ }^{a} P$-values were obtained using the Mann-Whitney $U$-test.

induced irregularities in the superficial and deep vascular plexus. OCT angiograms showed petaloid non-reflective area located mainly inside of the deep vascular plexus. We observed perifoveal microvascular changes similar to telangiectasia. The analysis of the choriocapillaris, and Sattler's and Haller's layers did not reveal findings of interest.

\section{Discussion}

We assessed the features of the inner/outer retina and the choroid in XLRS patients. Our main findings were that BCVA did not correlate with either CMT or SFCT in these patients. These results are consistent with previous reports indicating that BCVA remains stable if no secondary event (vitreous hemorrhage or retinal detachment) occurs. ${ }^{8-10}$ However, a recent study found that increased inner retinal foveal thickness and decreased perifoveal inner retinal thickness correlate with worse BCVA. ${ }^{7}$

In two previous pathology-based studies, inner retinal abnormalities were the most common finding in XLRS. ${ }^{11,12}$ However, other studies have shown that outer retinal abnormalities are also prevalent in this disorder. ${ }^{4,6,7,9,10}$ Our findings support the results of these previous reports. We found that the main manifestations of schisis were at the INL (89\%), OPL (72\%), and ONL $(61 \%)$. GCL was affected in only $17 \%$ of eyes. Our findings closely parallel those reported by Andreoli et al, ${ }^{7}$ who found that schisis affected the INL and OPL, respectively, in $85 \%$ and $61 \%$ of cases.
On SS-OCT, ELM and EPIS defects were present in more than two-thirds of eyes ( $72 \%$ and $67 \%$, respectively), while COST defects were observed in just over half (55\%) of the eyes. Damage in the outer retinal layers-mainly ELM, EPIS, and COST_correlated significantly with worse BCVA. Menke et al ${ }^{5}$ observed that retinal morphologic changes assessed with OCT did not correlate with BCVA; however, an important limitation of that study was the use of time-domain OCT for retinal imaging. Several studies have reported that abnormalities in the outer retinal layers occurring in various different scenarios (eg, epiretinal membrane, retinal detachment, and macular hole surgery) correlate significantly with worse visual acuity. ${ }^{13-16}$

To our knowledge this is the first report to evaluate retinal and choroidal thicknesses in patients with XLRS using the topographic map with nine subfields as defined by the ETDRS-style grid. We found that retinal and choroidal thicknesses did not differ significantly between the right and left eyes. Previous studies have described progressive changes in foveal thickness over time. ${ }^{10,17,18}$ Older patients tend to have fewer cystic changes and more frequently present an atrophic, hyperreflective appearance in OCT images. In advanced stages, the schisis collapses completely and intraretinal cysts disappear. ${ }^{5}$ Apparently, the retina may remain structurally stable in adolescence and atrophy may not appear until decades later. ${ }^{8,10}$

The median SFCT values were $305 \mu \mathrm{m}$ in OD and $307 \mu \mathrm{m}$ in OS in our sample (median age, 13 years). By comparison, Yang et al ${ }^{19}$ reported a median SFCT of $\sim 358 \mu \mathrm{m}$ (median age, 7.5 years; range 5-11), which was 
Table 3 Optical coherence tomographic features of patients with X-linked retinoschisis

\begin{tabular}{|c|c|c|c|c|c|c|c|}
\hline $\begin{array}{l}\text { Patient no./ } \\
\text { eye }\end{array}$ & GCL & INL & $O P L$ & ONL & $\begin{array}{l}\text { ELM } \\
\text { defect }\end{array}$ & $\begin{array}{l}\text { EPIS } \\
\text { defects }\end{array}$ & $\begin{array}{l}\text { COST } \\
\text { defects }\end{array}$ \\
\hline \multicolumn{8}{|l|}{1} \\
\hline OD & $x$ & $x$ & $x$ & $x$ & $x$ & $\mathrm{x}$ & $\mathrm{x}$ \\
\hline OS & $x$ & $x$ & $x$ & $x$ & $\mathrm{x}$ & $x$ & $x$ \\
\hline \multicolumn{8}{|l|}{2} \\
\hline OD & 0 & $x$ & $x$ & $\mathrm{x}$ & $x$ & $\mathrm{x}$ & $\mathrm{x}$ \\
\hline OS & 0 & $\mathrm{x}$ & $\mathrm{x}$ & 0 & 0 & 0 & 0 \\
\hline \multicolumn{8}{|l|}{3} \\
\hline OD & 0 & 0 & 0 & 0 & 0 & 0 & 0 \\
\hline OS & 0 & 0 & 0 & 0 & 0 & 0 & 0 \\
\hline \multicolumn{8}{|l|}{4} \\
\hline OD & 0 & $x$ & 0 & 0 & $x$ & $x$ & $x$ \\
\hline OS & 0 & $x$ & 0 & 0 & 0 & 0 & 0 \\
\hline \multicolumn{8}{|l|}{5} \\
\hline OD & $x$ & $x$ & $x$ & $x$ & $x$ & $x$ & $x$ \\
\hline OS & 0 & $\mathrm{x}$ & 0 & $\mathrm{x}$ & $x$ & $\mathrm{x}$ & $x$ \\
\hline \multicolumn{8}{|l|}{6} \\
\hline OD & 0 & $x$ & $x$ & $\mathrm{x}$ & $x$ & $\mathrm{x}$ & $x$ \\
\hline OS & 0 & $\mathrm{x}$ & $x$ & $x$ & $x$ & $x$ & $x$ \\
\hline \multicolumn{8}{|l|}{7} \\
\hline OD & 0 & $x$ & $x$ & 0 & $x$ & $x$ & 0 \\
\hline OS & 0 & $x$ & $x$ & 0 & $x$ & $x$ & 0 \\
\hline \multicolumn{8}{|l|}{8} \\
\hline OD & 0 & $\mathrm{x}$ & $x$ & $x$ & $x$ & $x$ & $x$ \\
\hline OS & 0 & $\mathrm{x}$ & $x$ & $x$ & $x$ & $\mathrm{x}$ & $\mathrm{x}$ \\
\hline \multicolumn{8}{|l|}{9} \\
\hline OD & 0 & $x$ & $\mathrm{x}$ & $\mathrm{x}$ & $x$ & 0 & 0 \\
\hline OS & 0 & $x$ & $x$ & $x$ & 0 & 0 & 0 \\
\hline $\begin{array}{l}\text { Average } \\
(\%)\end{array}$ & 17 & 89 & 72 & 61 & 72 & 67 & 55 \\
\hline
\end{tabular}

Abbreviations: COST, cones outer segment tips line; EPIS, ellipsoid portion of the inner segments; GCL, ganglion cell layer; INL, inner nuclear layer; $\mathrm{OD}$, right eye; $\mathrm{ONL}$, outer nuclear layer; $\mathrm{OPL}$, outer plexiform layer; OS, left eye; RNFL, retinal nerve fiber layer.

$35 \mu \mathrm{m}$ thicker in eyes with XLRS compared to control eyes; however, this difference was not statistically significant $(P=0.084)$. Hu et al ${ }^{10}$ described similar findings with respect to the SFCT (mean, $351.4 \mu \mathrm{m}$ ). Although the median SFCT values in our study were lower than previous reports, most of the eyes ( $>60 \%$ ) presented SFCT values $>300 \mu \mathrm{m}$. In addition, three of the eyes in our study with SFCT $<300 \mu \mathrm{m}$ had developed a retinal detachment requiring PPV. Several studies report that SFCT may change after vitrectomy for epiretinal membrane, macular hole, and rhegmatogenous retinal detachment. ${ }^{20-22}$
In our study, the superior parafoveal, temporal parafoveal, and temporal perifoveal zones were the thickest quadrants in patients with XLRS, although without statistically significant differences comparing similar zones between eyes. Several studies conducted in healthy children have shown that the thickest choroid is located in the superior-temporal location and the thinnest in the inferior-nasal areas. ${ }^{23-28}$ We found a significant positive correlation between age and SFCT in these patients. This same positive correlation between SFCT and age has also been reported in healthy children. ${ }^{23,27-29}$ By contrast, however, other authors have reported that the choroid becomes thinner with age in healthy children. ${ }^{25,30,31}$ The reasons for these discrepancies are not clear and more research is needed to elucidate the true association between choroidal thickness and age in XLRS.

We found no correlation between the spherical equivalent and SFCT. In our study, we decided to use patients' spherical equivalent instead of axial length (AL) determinations because this value is more convenient to obtain. The dependence of choroidal thickness on AL has been evaluated previously. ${ }^{24,28-30}$ Read et al ${ }^{29}$ reported that SFCT was thinner in the eyes of myopic vs nonmyopic children. By contrast, Park and $\mathrm{Oh}^{24}$ did not find a statistically significant correlation between central SFCT and AL.

On the images obtained on en-face OCT, we observed a spoke-like pattern in the foveal region as result of hyporeflective spaces at the INL and a reticular pattern in the parafoveal region. When the segmentation is performed at the level of OPL, we observed multiple polygonal hyporeflective cavities in the foveal region with hyperreflective partitions in the parafoveal region. We did not observe any characteristic pattern at the GCL on enface OCT; we had only two eyes with schisis at this level, but not extensive enough to evidence significant abnormalities. Yoshida-Uemura et al ${ }^{32}$ recently described (on SS-OCT) different schisis patterns in the various retinal layers; these patterns are highly consistent with our findings. Those authors observed several hyporeflective holes in the parafoveal zone in the GCL, a spoke-like pattern in the foveal region with a reticular pattern in the parafoveal zone when the segmentation was applied at INL. The schisis pattern at the OPL revealed multiple hyporeflective polygonal cavities in the foveal region with hyperreflective partitions in the parafoveal region in the OPL. These different patterns might be explained by variations in the distribution of cells in each retinal layer in the foveal and parafoveal regions, including bipolar, amacrine, horizontal, and Müller cells.

On OCT-A, some irregularities were seen at the superficial and deep vascular plexus (more prominent) 


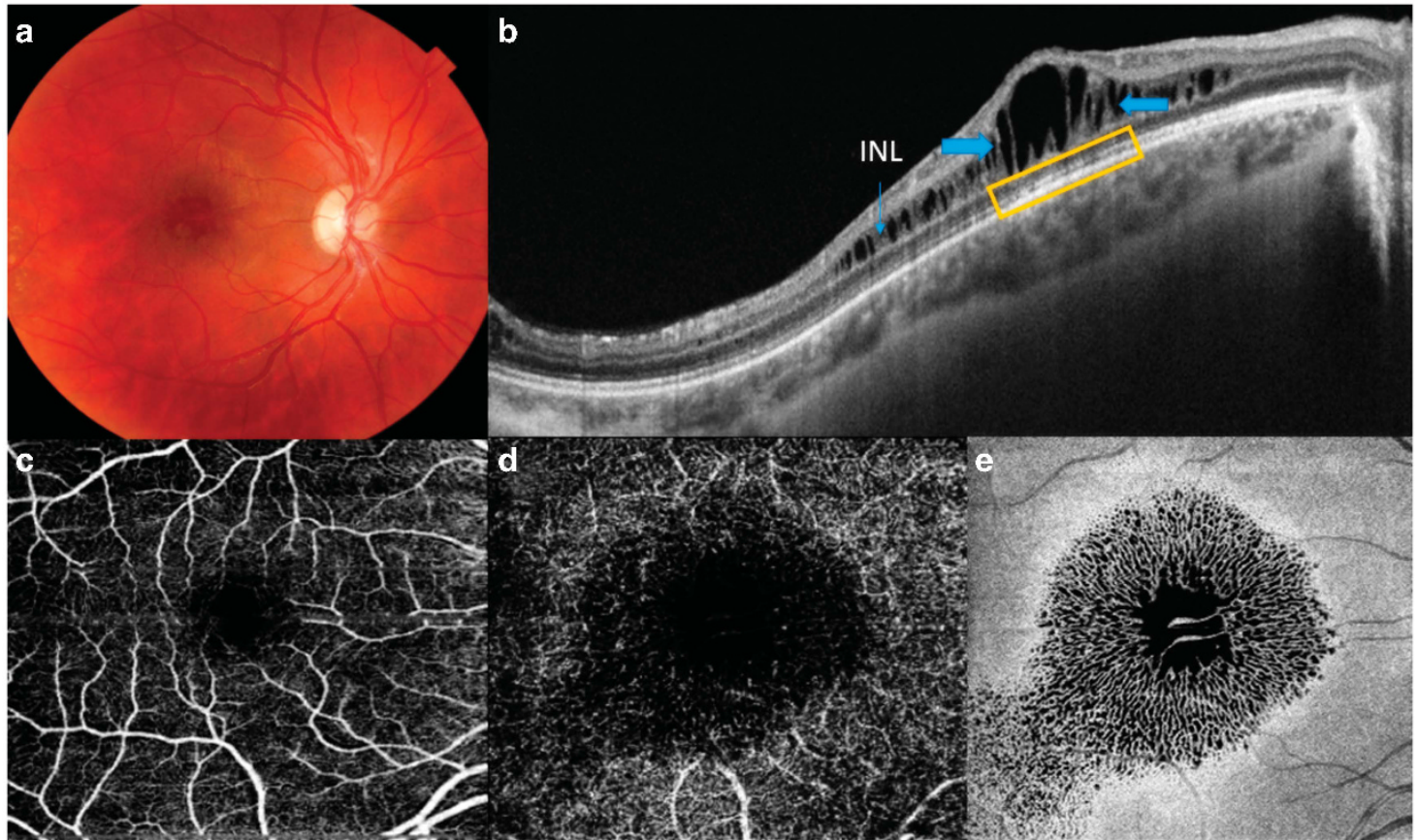

Figure 1 (a-e) The images correspond to a 13-year-old male patient (case No. 2) with XLRS. (a) Fundus color photography of the OD showing hyporeflective cystic spaces in the foveal and parafoveal regions. (b) SS-OCT of the OD revealing schisis (thick blue arrows) at the level of the INL (thin blue arrow). These images also show a small defect at the ELM, the EPIS ellipsoid zone, and COST (yellow rectangle). The upper/left (retinal) and lower/right (choroidal) areas show the topographic map with nine subfields as defined by the ETDRS-style grid. (c, d) A $6 \times 6 \mathrm{~mm}$ OCT-A showing superficial and deep vascular plexus (more prominent) with irregularities due to schisis at INL. OCT angiograms showed petaloid non-reflective area located predominantly inside of the deep vascular plexus. (e) An en-face OCT image at the level of the INL showing a spoke-like pattern in the foveal region and a reticular pattern in the parafoveal region.

due to the presence of schisis at the INL. OCT angiograms showed petaloid non-reflective areas located predominantly at the level of the deep vascular plexus and microvascular alterations similar to telangiectasia were observed in perifoveal area. Vascular alterations have been described in patients with XLRS, including Coats disease-like exudative retinopathy, dendritiform vessels in the retinal periphery, and perivascular sheathing. ${ }^{33}$ The analysis of the choriocapillaris did not evidence characteristics of any known vascular disorders. Stanga $e a^{34}$ have described the same petaloid pattern inside of the deep vascular plexus and perifoveal microvascular changes on OCT-A. They also reported areas of hypo- and hyper-reflectivity at the level of choriocapillaris that were not associated with any other vascular disorder but might be attributed to alterations in OCT-A signal due to RPE irregularities.

OCT-A is a promising, safe, non-invasive, and shortacquisition-time new technique that may be useful for the examination of the retinal and choroidal vasculature in children who are old enough to cooperate. Before the OCT-A, the only way to get information about the retinal and choroidal vessels had been the fluorescein angiography (FA) and indocyanine angiography (ICG), respectively. In cases of XLRS, the cystic-like spaces do not show hyperfluorescence when performing FA. By contrast, ICG demonstrates the cystic-like spaces, although this modality is more invasive than OCT-A. A distinct pattern (hyperfluorescent stellate) in the macular area associated with radial lines of hypofluorescence can be observed on the early phase of ICG. ${ }^{35}$

\section{Strengths and limitations}

One of the main strengths of this study is the use of new technologies (SS-OCT and OCT-A) to describe tomographic features in patients with XLRS. The main limitations are the retrospective design and lack of a control group. Moreover, because this was not a longitudinal study, we were unable to determine retinal changes in patients over time. Finally, the sample size was small but given the rarity of XLRS, obtaining larger samples is difficult. 


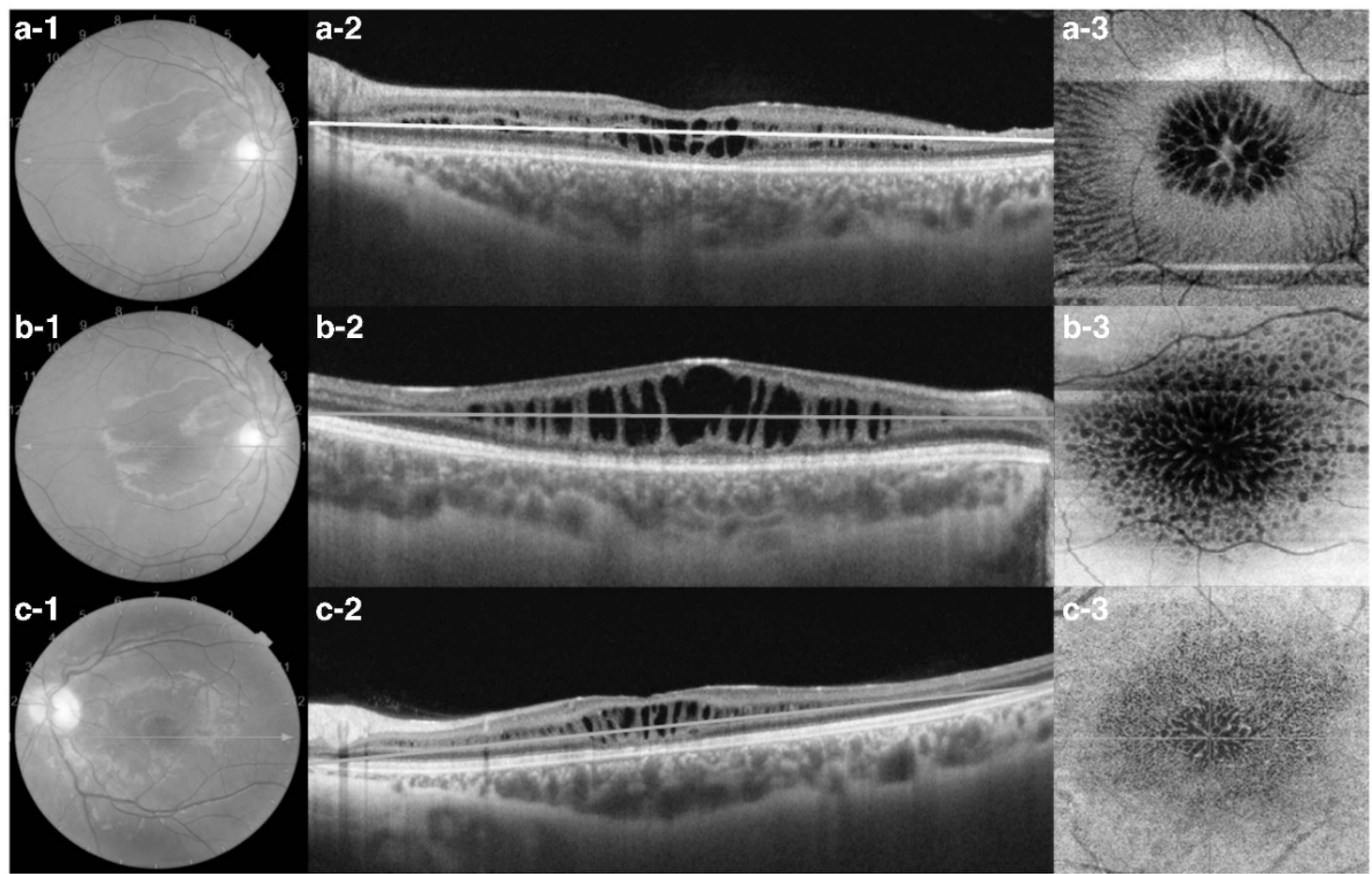

Figure 2 (a-1 to a-3) The images correspond to a 21-year-old male patient (case No. 9) with schisis at the level INL, OPL, and ONL in the OD. (a-1) Fundus color photograph shows maculopathy with a spoke-like appearance. (a-2) SS-OCT showing schisis at the INL, OPL, and ONL. The green line represents the segmentation at the border of the OPL (a-3). En-face OCT at the OPL showing multiple polygonal hyporeflective cavities in the foveal region with hyperreflective partitions in the parafoveal region. (b-1 to b-3) Fundus color photography and OCT images of a 16-year-old male (case No. 7) with schisis at the INL in OD. (b-2) SS-OCT showing hyporeflective cavities at the INL; the green line represents the segmentation. (b-3) En-face OCT showing a spoke-like pattern in the foveal region with reticular pattern in the parafoveal region. (c-1 to c-3) A 13-year-old boy with schisis at INL, OPL, and ONL in the OS. (c-1) Fundus color photography of the OS showing a spoke-like pattern. (c-2) SS-OCT revealing schisis at the INL, OPL, and ONL with segmentation at OPL (blue line). (c-3) En-face SS-OCT showing multiple polygonal hyporeflective cavities in the foveal region and hyperreflective partitions in the parafoveal region. A full colour version of this figure is available at the Eye journal online.

\section{Conclusions}

The hyporeflective spaces on SS-OCT were mainly observed at the INL and OPL. Although BCVA did not correlate with CMT and SFCT; ELM, EPIS, and COST defects were significantly correlated with worse BCVA. Retinal and choroidal thicknesses were not significantly different between eyes. The superior parafoveal, temporal parafoveal, and temporal perifoveal choroidal zones were the thickest quadrants. There was a positive correlation between age and SFCT. A spoke-like pattern in the foveal region and a reticular pattern in the parafoveal region were observed on en-face OCT when segmentation was performed at INL. In eyes in which schisis affected the OPL, multiple polygonal hyporeflective cavities were seen in the foveal region and hyperreflective partitions in the parafoveal region. SS-OCT and OCT-A are newer technologies that should be used routinely in patients with XLRS, the evaluation of inner/outer retina and choroid provide useful information that allow to understand better this uncommon disease.

\section{Summary}

What was known before

- Previous reports have evaluated the splitting of the ganglion cell and nuclear layers in patients with XLRS using optical coherence tomography (OCT). External limiting membrane, ellipsoid portion of the inner segments/inner segment outer segment junction line, cones outer segment tips line/interdigitation zone, and choroidal thickness have been also evaluated to explain the progressive visual loss in these patients. However, the evaluation of inner/outer retina and choroid characteristics with the use of newer technologies as swept-source (SS-OCT) and OCT angiography is very limited. SS-OCT provides faster and higher-quality scans than conventional spectral domain OCT and these advantages allow to obtain useful information to understand better an uncommon disease as XLRS.

What this study adds

- The hyporeflective spaces on SS-OCT were mainly observed at the INL and OPL. Although BCVA did not correlate with CMT and SFCT; ELM, EPIS, and COST defects were significantly correlated with worse BCVA. The superior parafoveal, temporal parafoveal, and 
temporal perifoveal choroidal zones were the thickest quadrants. There was a positive correlation between age and SFCT. A spoke-like pattern in the foveal region and a reticular pattern in the parafoveal region were observed on en-face OCT when segmentation was performed at INL. In eyes in which schisis affected the OPL, multiple polygonal hyporeflective cavities were seen in the foveal region and hyperreflective partitions in the parafoveal region. OCT angiography showed petaloid non-reflective areas located predominantly at the level of the deep vascular plexus and microvascular alterations similar to telangiectasia were observed in perifoveal area. The analysis of the choriocapillaris, and Sattler's and Haller's layers did not reveal findings of interest.

\section{Conflict of interest}

The authors declare no conflict of interest.

\section{Acknowledgements}

We thank Bradley Londres for editing and improving the manuscript. This work was supported by rarecommons. org.

\section{References}

1 Molday RS, Kellner U. Weber BHF. X-linked juvenile retinoschisis: clinical diagnosis, genetic analysis, and molecular mechanisms. Prog Retin Eye Res 2012; 31(3): 195-212.

2 Sauer CG, Gehrig A, Warneke-Wittstock R, Marquardt A, Ewing CC, Gibson A et al. Positional cloning of the gene associated with X-linked juvenile retinoschisis. Nat Genet 1997; 17(2): 164-170.

3 Audo I, Sahel JA, Mohand-Saïd S, Holder G, Moore A. X-linked retinoschisis. In: Querques G, Souied EH. Macular dystrophies. Switzerland: Springer, 2016 p. 71-81.

4 Yu J, Ni Y, Keane PA, Jiang C, Wang W, Xu G. Foveomacular schisis in juvenile $\mathrm{X}$-linked retinoschisis: an Optical Coherence Tomography Study. Am J Ophthalmol 2010; 149(6): 973-978.

5 Menke MN, Feke GT, Hirose T. Effect of aging on macular features of $x$-linked retinoschisis assessed with optical coherence tomography. Retina 2011; 31(6): 1186-1192.

6 Leng T. Two cases of X-linked retinoschisis with different spectral domain optical coherence tomography findings. Clin Ophthalmol 2012; 6: 1563-1565.

7 Andreoli MT, Lim JI. Optical coherence tomography retinal thickness and volume measurements in X-linked retinoschisis. Am J Ophthalmol 2014; 158(3): 567-573.

8 Kjellström C, Vijayasarathy V, Ponjavic P, Sieving A, Andréasson S. Long-term 12 year follow-up of X-linked congenital retinoschisis. Ophthalm Genet 2010; 31(3): 114-125.

9 Apushkin MA, Fishman GA, Janowicz MJ. Correlation of optical coherence tomography findings with visual acuity and macular lesions in patients with X-linked retinoschisis. Ophthalmology 2005; 112(3): 495-501.
10 Hu QR, Huang LZ, Chen XL, Xia HK, Li TQ, Li XX. Linked retinoschisis in juveniles: follow up by optical coherence tomography. Biomed Res Int 2017; 2017; 1704623.

11 Manschot WA. Pathology of hereditary juvenile retinoschisis. Arch Ophthalmol 1972; 88(2): 131-138.

12 Yanoff M, Kertesz Rahn E, Zimmerman LE. Histopathology of juvenile retinoschisis. Arch Ophthalmol 1968; 79(1): 49-53.

13 Shimozono M, Oishi A, Hata M, Matsuki T, Ito S, Ishida K et al. The significance of cone outer segment tips as a prognostic factor in epiretinal membrane surgery. Am J Ophthalmol 2012; 153(4): 698-704.

14 Park DH, Choi KS, Sun HJ, Lee SJ. Factors associated with visual outcome after macula-off rhegmatogenous retinal detachment surgery. Retina 2017 doi: 10.1097/ iae.0000000000001512. [epub ahead of print].

15 Kawashima Y, Uji A, Ooto S, Hangai M, Hosoda Y, Yoshimura N. Association between insufficient photoreceptor layer plugging and postoperative visual outcome in the surgically closed macular hole. Am J Ophthalmol 2015; 160(5): 982-989.

16 Landa G, Gentile RC, Garcia PM, Muldoon TO, Rosen RB. External limiting membrane and visual outcome in macular hole repair: spectral domain OCT analysis. Eye 2012; 26(1): 61-9.

17 The Retinoschisis Consortium. Functional implications of the spectrum of mutations found in 234 cases with X-linked juvenile retinoschisis. Hum Mol Genet 1998; 7(7): 1185-1192.

18 Takada Y, Fariss RB, Tanikawa A, Zeng Y, Carper D, Bush R et al. A retinal neuronal developmental wave of retinoschisin expression begins in ganglion cells during layer formation. Invest Ophthalmol Vis Sci 2004; 45(9): 3302-3312.

19 Yang HS, Lee JB, Yoon YH, Lee JY. Correlation between spectral-domain OCT findings and visual acuity in X-linked retinoschisis. Invest Ophthalmol Vis Sci 2014; 55(5): 3029-3036.

20 Kang EC, Lee KH, Koh HJ. Changes in choroidal thickness after vitrectomy for epiretinal membrane combined with vitreomacular traction. Acta Ophthalmol 2017; 95(5): e393-e398.

21 Bardak H, Gunay M, Bardak Y et al. Retinal and choroidal thicknesses measured with swept-source optical coherence tomography after surgery for idiopathic macular hole. Eur J Ophthalmol 2017; 27(3): 312-318.

22 Sayman Muslubas I, Karacorlu M, Hocaoglu M, Arf S, Uysal O. Subfoveal choroidal thickness change after pars plana vitrectomy in recent onset rhegmatogenous retinal detachment. Retina 2016; 36(12): 2371-2376.

23 Read SA, Collins MJ, Vincent SJ, Alonso-Caneiro D. Choroidal thickness in childhood. Invest Ophthalmol Vis Sci 2013; 54(5): 3586-3593.

24 Park KA, Oh SY. Choroidal thickness in healthy children. Retina 2013; 33(9): 1971-1976.

25 Nagasawa T, Mitamura Y, Katome T, Shinomiya K, Naito T, Nagasato D et al. Macular choroidal thickness and volume in healthy pediatric individuals measured by swept-source optical coherence tomography. Invest Ophthalmol Vis Sci 2013; 54(10): 7068-7074.

26 Ruiz-Moreno JM, Flores-Moreno I, Lugo F, Ruiz-Medrano J, Montero JA, Akiba M. Macular choroidal thickness in normal pediatric population measured by swept-source optical coherence tomography. Invest Ophthalmol Vis Sci 2013; 54(1): 353-359.

27 Bidaut-Garnier M, Schwartz C, Puyraveau M, Montard M, Delbosc B, Saleh M. Choroidal thickness measurement in 
children using optical coherence tomography. Retina 2014; 34 (4): 768-774

28 Herrera L, Perez-Navarro I, Sanchez-Cano A, Perez-Garcia D, Remon L, Almenara C et al. Choroidal thickness and volume in a healthy pediatric population and its relationship with age, axial length, ametropia, and sex. Retina 2015; 35(12): 2574-83.

29 Read SA, Collins MJ, Vincent SJ, Alonso-Caneiro D. Choroidal thickness in myopic and nonmyopic children assessed with enhanced depth imaging optical coherence tomography. Invest Ophthalmol Vis Sci 2013; 54(12): 7578-7586.

30 Fujiwara A, Shiragami C, Shirakata Y, Manabe S, Izumibata S, Shiraga F. Enhanced depth imaging spectral-domain optical coherence tomography of subfoveal choroidal thickness in normal Japanese eyes. Jpn J Ophthalmol 2012; 56(3): 230-235.

31 Ruiz-Medrano J, Flores-Moreno I, Peña-García P, Montero JA, Duker JS, Ruiz-Moreno JM. Macular choroidal thickness profile in a healthy population measured by swept-source optical coherence tomography. Invest Ophthalmol Vis Sci 2014; 55(6): 3532-3542.

32 Yoshida-Uemura T, Katagiri S, Yokoi T et al. Different foveal schisis patterns in each retinal layer in eyes with hereditary juvenile retinoschisis evaluated by en-face optical coherence tomography. Graefes Arch Clin Exp Ophthalmol 2017; 255(4): 719-723.

33 Kim DY, Mukai S. X-linked juvenile retinoschisis (XLRS): a review of genotype-phenotype relationships. Semin Ophthalmol 2013; 28(5-6): 392-6.

34 Stanga PE, Papayannis A, Tsamis E, Chwiejczak K, Stringa F, Jalil A et al. Swept-source optical coherence tomography angiography of paediatric macular diseases. Dev Ophthalmol 2016; 56: 166-73.

35 Souied EH, Goritsa A, Querques G, Coscas G, Soubrane G. Indocyanine green angiography of juvenile $X$-linked retinoschisis. Am J Ophthalmol 2005; 140(3): 558-61. 